\title{
On Mobile Sensor Assisted Field Coverage
}

DAN WANG

\author{
The Hong Kong Polytechnic University \\ JIANGCHUAN LIU \\ Simon Fraser University \\ and
}

QIAN ZHANG

Hong Kong University of Science and Technology

Providing field coverage is a key task in many sensor network applications. With unevenly distributed static sensors, a quality coverage with acceptable network lifetime is often difficult to achieve. Fortunately, the recent advances on embedded and robotic systems make mobile sensors possible, and we suggest that a small set of mobile sensors can leveraged toward a cost-effective solution for field coverage. There are however a series of fundamental questions to be answered in such a hybrid networks of static and mobile sensors: 1) Given the expected coverage quality and system lifetime, how many mobile sensors should be deployed? 2) What are the necessary coverage contributions from each type of sensors? and 3) What working and moving patterns should the sensors adopt to achieve the desired coverage contributions?

In this paper, we offer an analytical study on the above problems, and the results also lead to a practical system design. Specifically, we present an optimal algorithm for calculating the contributions from different types of sensors, which fully exploits the potentials of the mobile sensors and maximizes the network lifetime. We then present a random walk model for the mobile sensors. The model is distributed with very low control overhead. Its parameters can be finetuned to match the moving capability of different mobile sensors and the demands from a broad spectrum of applications. A node collaboration scheme is then introduced to further enhance the system performance.

We demonstrate through analysis and simulation that, in our mobile assisted design, a small set of mobile sensors can effectively address the uneven distribution of the static sensors and significantly improve the coverage quality.

Categories and Subject Descriptors: C.2.2 [Computer-Communication Networks]: Network Protocols-application; F.2.2 [Analysis of Algorithms and Problem Complexity]: General

General Terms: Algorithms, Analysis, Design, Experimentation

Additional Key Words and Phrases: Wireless sensor networks, Sensor coverage, Random walk

Author's address: D. Wang, Department of Computing, The Hong Kong Polytechnic University, Hung Hom, Kowloon, Hong Kong, Email: csdwang@comp.polyu.edu.hk. J. Liu, School of Computing Science, Simon Fraser University, Burnaby, BC, Canada, V5A 1S6, Email: jcliu@cs.sfu.ca, Q. Zhang: Department of Computer Science and Engineering, Hong Kong University of Science and Technology, Clear Water Bay, Kowloon, Hong Kong, Email: qianzh@cse.ust.hk.

A preliminary version of this paper is in proceeding of the 15th IEEE International Workshop on Quality of Service (IWQoS'07), Evanston, IL, June 2007.

Permission to make digital/hard copy of all or part of this material without fee for personal or classroom use provided that the copies are not made or distributed for profit or commercial advantage, the ACM copyright/server notice, the title of the publication, and its date appear, and notice is given that copying is by permission of the ACM, Inc. To copy otherwise, to republish, to post on servers, or to redistribute to lists requires prior specific permission and/or a fee.

(C) 2010 ACM $1529-3785 / 2010 / 0700-0111 \$ 5.00$ 


\section{INTRODUCTION}

Wireless sensor networks have recently been suggested for many protection and surveillance applications. One key objective of these applications is to detect abnormal events in a sensing field, which depends on the coverage quality of the sensor network. The $k$-coverage is a common criterion, where any point in the sensor field should be covered by $k$ sensors [Slijepcevic and Potkonjak 2001]. For many applications, it turns out that a deterministic $k$-coverage is too expensive and not necessary. Therefore, probabilistic coverage [Gui and Mohapatra 2004][Xu et al. 2001 is introduced and every point is covered with certain ratio. This ratio tunes the coverage quality and allows the sensors to switch between sleeping and working states.

In these studies, only static sensors are used. The quality of coverage is noticeably affected by the initial deployment of the sensors. For uneven sensor distributions, the sensors in a sparse area may have to stay active longer to ensure the coverage quality. The batteries of these sensors will be depleted earlier and thus making the area even sparser. In the extreme case, an area will be uncovered by any sensor, leaving a hole in the field. Unfortunately, such unfavorable sensor distributions are inevitable in many applications where a well-controlled or manual deployment is not practical.

Recent advances of embedded hardware and robot have made mobile sensors possible. The mobile sensors have the same sensing capability as static sensors, but are able to move in a field, and their batteries can be rechargeable. In other words, their lifetime is not necessarily bounded by the limited battery. While fully mobile sensor networks remain expensive and the routing and information exchange can be quite complicated therein, we expect that a hybrid network assisted by a small set of mobile sensors can be a cost-effective solution toward coverage with unevenly distributed sensors. A first design in this direction was presented in [Wang et al. 2003], which suggested a reposition of the mobile sensors after the initial deployment. The one-time reposition unfortunately cannot well combat the uneven sensor distribution in many cases. Consider Fig. 1, where there are a number of static sensors and three mobile sensors to cover a field. Each sensor can cover its associated grid. If there are no mobile sensors, grid 6 will never be covered. If only one-time repositioning for the mobile sensors is employed, the coverage can be enhanced, but there will still remain grids with permanently fewer sensors than others.

In this paper we propose a mobile sensor assisted network that fully exploits the movement capability of the mobile sensors. In our solution, the mobile sensors are always in motion to assist the static sensors; the occurrence probability of the mobile sensors in each grid, or their contribution for covering the grid, is adaptively determined according to the network configuration. From a statistical point of view, the overall coverage is enhanced, and energy consumption of the static sensors is more balanced.

The main challenges in designing such a mobile sensor assisted network are, first, to clarify the necessary coverage contributions from the static and mobile sensors; and second, to find specific models for the mobile sensors to achieve their desired coverage contribution. In this paper, we for the first time offer an analyt- 


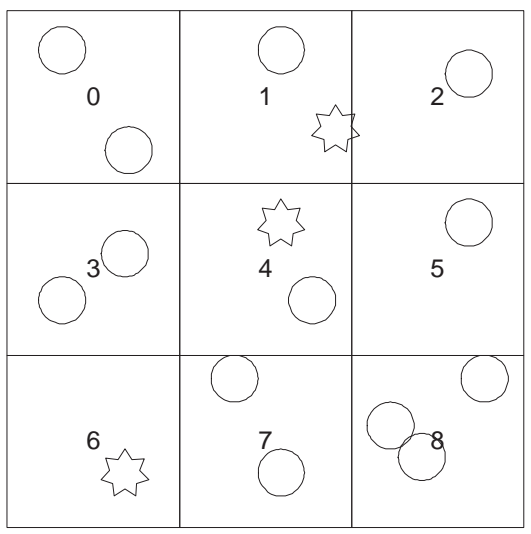

Fig. 1. Field covered by a mobile sensor assisted network, circles representing static sensors and stars representing mobile sensors.

ical study on the above problems, and the results also lead to a practical system design. Specifically, we present an optimal algorithm for calculating the contributions, which fully explores the potentials of the mobile sensors and maximizes the network lifetime. We also illustrate the number of mobile sensors needed given the expected system lifetime and the coverage quality requirement. We show concrete schemes to achieve the contributions. For the static sensors, we use a simple random sleep/work scheduling. We then present a random walk model for the mobile sensors that achieves their coverage contribution.

Our mobile sensor assisted architecture is general enough and offers a promising baseline for the demands from diverse applications. Various enhancements can be integrated to improve the performance of the system. Indeed, we point out several interesting observations from this design. Particularly, a wall effect may prevent mobile sensors from moving freely in a field. We effectively solve this problem through an optimal mobile sensor allocation algorithm. We then outline a sensor collaboration scheme which further reduces redundant coverage from different sensors.

Extensive simulations have been carried out to study our mobile sensor assisted network under various configurations. The results demonstrate that a small set of mobile sensors can significantly improves the coverage quality and the system lifetime.

The rest of the paper is organized as follows. In section 2, we present the related work. We outline our mobile sensor assisted architecture in section 3. The respective contributions from static and mobile sensors are derived in section 4. Section 5 discusses the random walk based mobility model and solutions for the wall effect. In section 6 , we present an in-network collaboration protocol to avoid redundant activation. The performance of the mobile sensor assisted network is evaluated in section 7 . We discuss a generalization of the underlying grid structure in section 8 . Finally, section 9 concludes the paper. 


\section{RELATED WORK}

Wireless sensor networks have been widely studied in recent years, focusing on those with static sensors; a survey can be found in [Akyildiz et al. 2002]. The effective coverage using static sensors is one of the key problems in sensor network applications, and has been examined in various aspects, such as field/path coverage and determinstic/probabilistic coverage. Related work can be found in [Gui and Mohapatra 2004][Slijepcevic and Potkonjak 2001][Yan et al. 2003] and the references therein. Many studies propose grouping the sensors into grids [Gui and Mohapatra 2004][Xing et al. 2004][Xu et al. 2001], where all sensors in a grid are equivalent in their functionality, such as coverage capability. The surveillance systems in [Gui and Mohapatra 2004][Yan et al. 2003] further suggest that the static sensors can be redundantly deployed and work in turn to extend the lifetime of the system. Especially, a collaborative protocol is built in PEAS [Ye et al. 2003], where the sensors exchange messages with the neighbor nodes. When a sensor observes that a certain number of neighbors are awake, it goes back to sleep. Similar collaborative schemes with different objectives can be found in [Gui and Mohapatra 2004][Wang et al. 2007]. These schemes are robust to node failures. However, they usually do not provide coverage guarantee. A probabilistic coverage scheme RIS is proposed in [Kumar et al. 2004], where the static sensors are randomly deployed and activate themselves with certain probability. It studies the relationship of such parameters as the total number of static sensors needed, the activation probability, the sensing range etc. Similar schemes with different objectives have been studied in [Yan et al. 2003][Wang et al. 2007]. The sensors in these schemes usually make independent decisions; and hard or statistical coverage guarantee can be achieved. As shown in the Introduction, using randomly deployed static sensors only may results in uneven load on each individual sensors. Therefore, our configurations for the static sensors are motivated by their work, but emphasize on the interactions with the mobile sensors.

The advances in embedded systems and hardware designs have realized mobile sensors, such as Robomote [Sibley et al. 2002] and Khapera [Mondada et al. 1993]. Unlike the static sensors, which are tightly constrained by the energy supplies, their batteries are rechargeable. Recent work also suggests that much longer working time and shorter recharging time can soon be expected [Kansal et al. 2004].

The mobility model of mobile nodes has long been a classic problem in ad hoc and cellular wireless network research. The random walk, random waypoint walk, random trip, and fluid models have been widely used to capture the mobile behaviors. A survey and comparison of these models can be found in [Schindelhauer 2006]. However, most of them analyze the mobility behaviors, while not for guiding the movement of the mobile nodes.

Using mobile sensors for coverage has been recently considered in [Liu et al. 2005][Wang et al. 2003]. Liu et al. [Liu et al. 2005] extend the definition of coverage, which is originally given in static geographic sense, into the time domain. Informally, the coverage is evaluated as the fraction of the covered area at a point of time. They conclude that, compared to using uniformly distributed static sensors, it is more beneficial if all sensors are mobile and are traveling in a random walk fashion. A more recent work [Bisnik et al. 2006] studies the velocity and movement 
strategies for a network of mobile sensors to improves the field coverage. It shows the relationship of the necessary number of mobile sensors, the minimum speed and the event detection (coverage) requirement. However, energy is not considered in their work. While these theoretical results are elegant and exciting, the mobile sensors remain expensive nowadays; it is unlikely a fully mobile sensor network is practical in the near future. In addition, when all the sensors are in random movement, packet routing (e.g., after an abnormal event is detected) and information dissemination will be much more complicated.

We thus envision a mobile sensor assisted network consisting of static and a few mobile sensors. If the number of the mobile sensors is small, the cost of building such a network remains acceptable, and the performance could be significantly improved, as shown in our study. A first study towards building a hybrid sensor network is presented in [Wang et al. 2003], which compensates poor initial sensor distributions by strategically repositioning some mobile sensors. Specifically, the sensors, after deployment, will first estimate the coverage holes according to a Voronoi diagram and then use a bidding protocol to guide the mobile sensors to better positions. The bidding protocol trade-offs the moving distance and the coverage quality. Another work is in [Zou and Chakrabarty 2004] where the new locations are calculated based on virtual force. More specifically, if the distance between two sensors is less than a threshold, there is a repulsive (negative) force to push them apart. Otherwise, there is a attractive (positive) force between them. The key difference here is that we consider continuous movement for the mobile sensors, while they focus on one-time repositioning. Some other one-time reposition schemes can be found in [Howard et al. 2002a][Howard et al. 2002b][Zou and Chakrabarty 2003] and a common drawback is that, after the mobile sensors are reposited, the field coverage may still be unbalanced, possibly leaving coverage holes. Our proposal can be viewed as a series repositioning of the mobile sensors. Over a long range of time, each grid will statistically have a better share of the presence (coverage) of the mobile sensors. We demonstrated the potential benefit of continuous movement through both analytical and experimental results.

\section{ARCHITECTURE OVERVIEW}

\subsection{Network Models}

The mobile sensor assisted network in our study consists of both static and mobile sensors, which collectively monitor a field of interest. As in previous studies [Gao et al. 2001][Karp and Kung 2000][Xu et al. 2001], we assume that the field is a square and can be divided into $n^{2}$ virtual grids, indexed from 0 to $n^{2}-1^{1}$. This virtual grid structure is not special, and we will show in Section 8 that our analysis and algorithms can be easily extended to hexagon or other virtual structures. We admit that a square field is a simplification. For any arbitrary field, a set of virtual square grids may not exact match the field especially in the borders. How to partition the field so that it can be covered by a set of grids falls in the scope of computational geometry. In this paper, we take this simplification the same as the previous works so that we can focus on the behavior of the sensor nodes. Through

\footnotetext{
${ }^{1}$ In this paper, we use the grids to denote a grid of $n^{2}$ cells.
} 


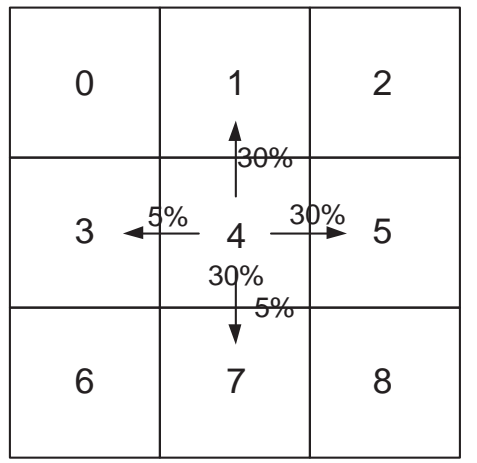

Fig. 2. The movement of a mobile sensor. The probabilities for moving to or staying in a grid are determined according to the network configuration.

GPS or available positioning services [Albowitz et al. 2001][Bulusu et al. 2000], the sensors are aware of their location information and, hence, their associated grids. The size of each grid is $\frac{\sqrt{2}}{2} R \times \frac{\sqrt{2}}{2} R$, where $R$ is the sensing range of a static sensor. As such, any active sensor in a grid can cover the whole grid. The sensing range of a mobile sensor can be smaller, e.g., $\frac{R}{2}$, as it can reposition itself to the center of its grid. An example of the grid structure is shown in Fig. 1.

Notice that in this paper, we only focus on sensing and coverage, when a sensor (mobile or static) detects an abnormal event in its grid, it should report the event to a predefined agent. The reporting mechanism is out of the scope of our study, and existing virtual grid based algorithms can be used [Xu et al. 2001].

Given that the static sensors in one grid are equivalent in coverage, they do not have to be active simultaneously, so as to save energy. The deployment of the static sensors is often nonuniform; and even worse, holes (grids with no static sensors) can exist, creating permanently uncovered regions ${ }^{2}$. On the other hand, the mobile sensors are always active, and can move in the field over time (Fig. 2), which we will explore to boost the coverage quality.

\subsection{Performance Measurement}

Since our focus is on field coverage, we define a measure of how well a location is covered as below, which is motivated by [Xing et al. 2004].

Definition 1. A sensor field is said to be $\delta$-covered if, at any point in time, at least an expected $\delta \in(0,1)$ fraction of the whole area is covered by one or more active sensors ${ }^{3}$.

Let $\delta$ be the minimum coverage ratio required by the user, our objective is to ensure this quality, while maximizing the lifetime of the network.

It is worth noting that the battery of state-of-the-art mobile sensors is rechargeable [Kansal et al. 2004]; hence, the lifetime of the whole network is bounded by

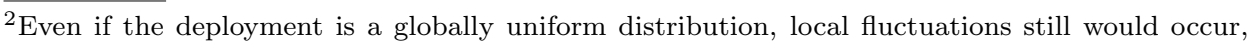
resulting in uneven numbers of sensors in different grids.

${ }^{3}$ Notice that in this definition, we are more restricted as we request in every point of time, the expected coverage is above $\delta$.

ACM Transactions on Computational Logic, Vol. V, No. N, October 2010. 
that of the static sensors. As a measurement, we use the lifetime of the first dying out sensor to represent the system lifetime. This definition has been widely used in existing studies [Chang and Tassiulas 2000][Younis and Fahmy 2004], and essentially suggests a load-balanced operation for the static sensors. The effectiveness of this definition has been validated by our simulation results in Section 7 . From a functional point of view, once the first static sensor dies, its grid needs additional assistance from the mobile/static sensors, which in turn increases the workload of other static sensors, resulting in a domino effect that quickly drains the power of the whole network. Thus, the death of the first sensor serves as a good indication to the end of the steady-state operation. Note that, however, our architecture is general enough that other load balancing lifetime definitions are possible. For example, the system lifetime is equal to the expected lifetime of a static sensor, or the expected working time of a static sensor with $10 \%$ of its residual energy.

In summary, given a coverage requirement, the network lifetime depends on the activation models of the static sensors, which further depend on the sensor distribution and the potential contributions from the mobile sensors.

\subsection{Working and Moving Models}

Given the system model and the performance measures, a natural question is what kind of working and moving models of the sensors can achieve the coverage objective. In our basic framework, we adopt a random activation scheduling for the static sensors, and a random walk model for the mobile sensors. More specifically, our mobile sensor assisted network goes through the following stages:

1) Parameter Initialization: After deployment, one or more mobile sensors travel around the field and collect the distribution information of the static senors in all grids. The mobile sensors compute the movement schemes for themselves (algorithms to be specified in Section 5) as well as the activation probability of the static sensors (algorithms to be specified in Section 4). The mobile sensors then notify the static sensors of their activation probability.

2) Field Monitoring: Consider the time slots are discrete. In each time slot, a static sensor independently activates itself with the activation probability obtained in the initialization stage and then monitors its grid. Each mobile sensor independently decides to move into one neighboring grid or to stay in the current grid, and then monitors the grid where it resides.

The advantages of using a probabilistic operation over a deterministic one are many. First, our technique is easier to implement because it involves simple optimization in the initial stage for the sensors. Second, the behavior of each type of the sensors are statistically identical. This is useful especially for recharging or replacement of mobile sensors. The substitute mobile sensors can easily follow the mobility model and continue to monitor the sensor field, regardless of the current state of other sensors, whereas a deterministic scheme may involve re-optimization. Third, a probabilistic coverage is generally more resistent to intruders that try to learn the sensor behaviors.

Our architecture offers achievable and reasonably good solutions to the problem of the uneven distribution of static sensors. It is however worth emphasizing that many practical enhancements could be added to this baseline framework, and we will discuss some of them later. 


\begin{tabular}{|c|l|}
\hline Notation & Definition \\
\hline$n$ & Grid dimension \\
$p$ & Total number of grids, i.e., $n^{2}$ \\
$R$ & Activation probability for static sensors \\
$\delta$ & Rensing range of a static sensor \\
$d(i)$ & Dequired coverage ratio for the sensor field \\
$l_{i}$ & The index of grid with density rank $i$ \\
$M$ & Number of mobile sensors \\
$P_{i j}$ & Probability that a mobile sensor moves from grid $i$ to $j$ \\
$\pi_{j}$ & Coverage contribution by a mobile sensor for grid $j$ \\
$\pi$ & Vector of $\pi_{i}$ \\
$m_{i}$ & Mobile sensor $i$ \\
$s_{i}$ & Static sensor $i$ \\
\hline
\end{tabular}

Table I. List of Notations

For ease of exposition, we list the notations used in this paper in Tab. I.

\subsection{Performance at a Glance}

Before going into the detail design, we first make an analytical comparison of the performance of three different strategies, i.e., using static sensors only, mobile sensors with one-time reposition and our mobile assisted scheme. Let the number of grids be $N$; the number of mobile sensor nodes be $M$; and the number of static sensor nodes be $\mathcal{N}$. Assume to obtain an expected system lifetime span, the activation probability of a static sensor node should be less than $\hat{p}$.

1) Static Sensors vs. One-Time Reposition: We compare the number of static sensors needed to achieve the required coverage ratio $\delta$ by these two schemes.

We first consider the minimum density of a grid if there are only static sensors. Let the density of static sensors in a grid be $d$. To achieve the required coverage ratio $\delta$, we should have $(1-\hat{p})^{d} \leq 1-\delta$; which implies $d \geq \frac{\log (1-\delta)}{\log (1-\hat{p})}$. Let $Y_{i}$ denote a random variable of sensor node $i$ such that $Y_{i}=1$ if node $i$ falls in a certain grid, and $Y_{i}=0$ otherwise. Then, $\operatorname{Pr}\left[Y_{i}\right]=\frac{1}{N}$. As we assume the sensor nodes are uniformly and randomly deployed, consequently, $Y_{0}, Y_{1}, \ldots, Y_{\mathcal{N}}$ are independent. Let $Y=\sum_{i=0}^{\mathcal{N}} Y_{i}$. By Chernoff Bound, we have $\operatorname{Pr}[Y<d]=\operatorname{Pr}\left[Y<\frac{d}{E[Y]} E[Y]\right] \leq$ $e^{-\left(1-\frac{d}{E[Y]}\right)^{2} \frac{E[Y]}{2}}$. Notice that here $E[Y]=\frac{\mathcal{N}}{N}$ and $\frac{d}{E[Y]}<1$ must be satisfied.

If we have static sensors only, to maintain the coverage, the expected number of grids that has less than $d$ static sensors must be less than 1. Therefore, $\operatorname{Pr}[Y<$ $d] \times N \leq 1$. This implies that $e^{-\left(1-\frac{\log (1-\delta) N}{\log (1-\hat{p}) N}\right)^{2} \times \frac{\mathcal{N}}{2 N}} \times N \leq 1$.

Then consider that we have mobile sensors and apply one-time reposition scheme. To simplify the analysis, we assume that there is a perfect movement scheme so that each mobile sensor is able to move to the right grid which has insufficient coverage. Since we have $M$ mobile sensors, to maintain the coverage, we need $\operatorname{Pr}[Y<d] \times N \leq M$. This implies that $e^{-\left(1-\frac{\log (1-\delta) N}{\log (1-\hat{p}) N}\right)^{2} \times \frac{\mathcal{N}}{2 N}} \times N \leq M$.

Thus, the number of static sensors needed is: 


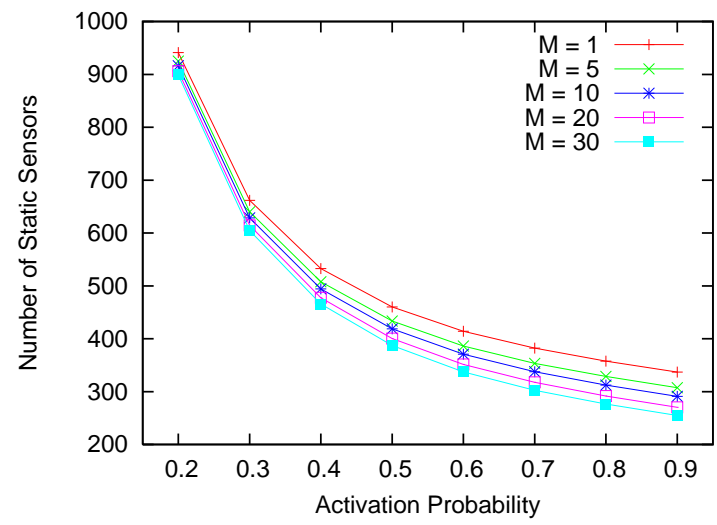

Fig. 3. The number of static sensors needed as a function of activation probability; $N=100$, $\delta=0.85$.

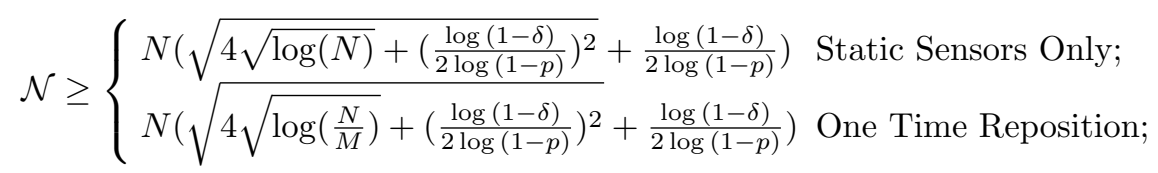

To better understand these two inequalities, we plot the numerical results shown in Fig. 3, where $M=1$ indicates the scheme of static sensors only. Not surprisingly, we can see that when the activation probability is low, the number of static sensors needed is high. Another important observation is that when the total number of static sensors is high $(\sim 900)$, the impact of increasing the number of mobile sensors (from 5 to 30 ) is much less significant than that when the total number of static sensors is low. This is not surprising as when the reposition is done, the mobile sensor is dedicated to a certain grid. Thus, if the number of static sensors is high, say, 900, the impact of 30 mobile sensors is much less than their impact upon 300 static sensor. We see that in [Wang et al. 2003], the ratio of the mobile sensors is $30 \%$, which is a large fraction of the network nodes. In this paper, we fully release the mobile sensors so that they do not need to dedicate to a certain grid and show that an even smaller number of mobile sensors can achieve better performance.

2) One-Time Reposition vs. Mobile Assisted Scheme: We then look into our Mobile Assisted Scheme. We study the number of mobile sensors that are needed to achieve the same coverage ratio $\delta$ given the total static sensors fixed. To simplify the analysis, we still assume that there is a perfect mobility scheme to fully utilize the potential of the mobile sensors.

Consider a specific grid in the mobile assisted scheme. If the number of static sensors in this grid is $k$, the total uncover probability is $(1-\hat{p})^{k}$. Let the assistance from the mobile sensors for this grid to be $\pi_{k}$. That is, the probability that a mobile sensor will be present is $\pi_{k}$. As the coverage requirement is $\delta$, we should have $(1-\hat{p})^{k} \times\left(1-\pi_{k}\right) \leq(1-\delta)$; which implies $\pi_{k} \geq 1-\frac{(1-\delta)}{(1-\hat{p})^{k}}$. The number of grids with $k$ static sensors is $(\operatorname{Pr}[Y<k]-\operatorname{Pr}[Y<k-1]) \times N$. The necessary number of mobile sensors for covering the grids with $k$ static sensors is thus $(\operatorname{Pr}[Y<$ 


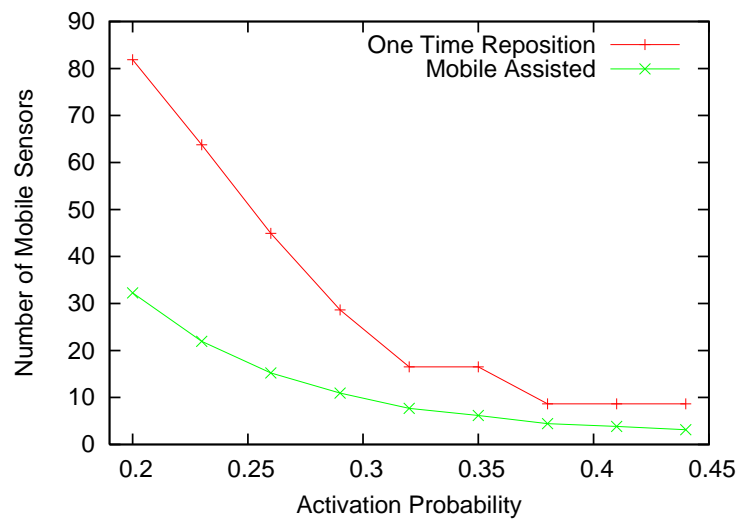

Fig. 4. The number of mobile sensors as a function of activation probability; $N=100, \mathcal{N}=1000$, $\delta=0.85$.

$k]-\operatorname{Pr}[Y<k-1]) \times N \times \pi_{k}$. Notice that no mobile sensor assistance is needed for the grids with $d$ static sensors where $d$ satisfies $(1-\hat{p})^{d} \geq(1-\delta)$, i.e., $d=\left\lceil\frac{\log (1-\delta)}{\log (1-\hat{p})}\right\rceil$.

For one-time reposition, each mobile node has to be fixed in a grid and the presence cannot be fractional. As a summary, the total number of mobile sensors needed in these two schemes are:

$$
M \geq \begin{cases}\left(\sum_{i=1}^{d}(\operatorname{Pr}[Y<i]-\operatorname{Pr}[Y<(i-1)])\right) \times N & \text { One Time Reposition } \\ \left(\sum_{i=1}^{d}\left((\operatorname{Pr}[Y<i]-\operatorname{Pr}[Y<(i-1)]) \pi_{i}\right)\right) \times N & \text { Mobile Assisted }\end{cases}
$$

We plot the numerical results in Fig. 4. Not surprisingly, we can see that the Mobile Assisted scheme performs better than the One-Time Reposition scheme. We can see that when the activation probability of static sensors is $p=0.2$, the number of mobile sensors needed for one-time reposition is 2.5 times than that of the mobile assisted scheme. When the activation probability of the static sensors is high, the performance of the mobile assisted scheme is not as significant in the figure. The reason is that the above analysis is based on Chernoff Bound. The original form of Chernoff Bound is $\operatorname{Pr}[Y<(1-\epsilon) \mu]<e^{-\frac{\epsilon^{2} \mu}{2}}$. Here $0<\epsilon<1$ and Chernoff Bound has higher precision when $\epsilon$ is small. In our analysis, this means that $0<\frac{d}{E[Y]}<1$ and the smaller $\frac{d}{E[Y]}$ is, the tighter the bound is. Easily, we see that the bound is tighter when $p$ is small.

Nevertheless, these analytical analysis has shown us the advantage of our mobile assisted scheme. We will develop specifically the contribution from different types of sensors, a concrete movement scheme for the mobile sensors, as well as the simulations in the following sections.

3) Energy Comparison: The advantage of mobile assisted scheme does not come for free. The mobile sensors need to move continuously, which consumes energy. It has been shown that Robomote consumes 27.96 Joule per meter [Sibley et al. 2002]. The communication is usually in the order of $100 \times 10^{-9}$ Joule per bit [Heinzelman et al. 2000]; and the sensing is even an order less energy consuming ACM Transactions on Computational Logic, Vol. V, No. N, October 2010. 


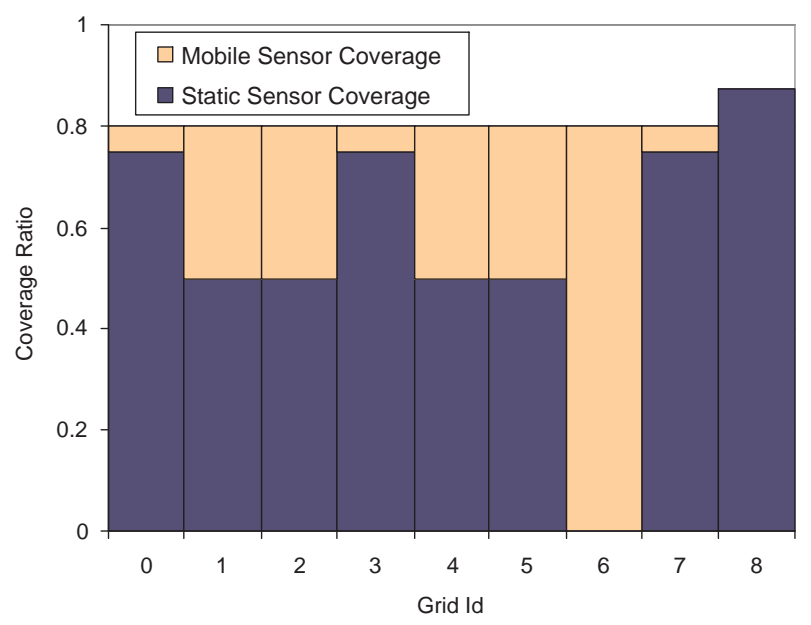

Fig. 5. Coverage contributions from static and mobile sensors. Coverage ratio $\delta=0.8$, and activation probability of static sensors $p=0.5$.

than communication. While the energy consumption of the sensors is well-known to vary based on the device and the functionality, one may analyze a trade-off point where our scheme best fits in. We emphasize, however, that the mobile sensors are more easily to move to certain locations (e.g., a charging dock) to re-charge their batteries or to have them being replaced. This is particularly important for applications in harsh environments, where battery recharge or change is difficult for static sensors. Examples include chemical plant monitoring where the safety of human technician entrance is not known, or habitat monitoring where the birds are sensitive to human intervention. With mobile sensors, the human technicians can stay within a distance waiting for them to return for battery re-charge or change.

We consider it future work where the traveling distance of the mobile sensors should be joint optimized for those applications where the sensor batteries are not easy to recharge and the energy reserve of the mobile sensors is within a range to that of the static sensors.

\section{COVERAGE CONTRIBUTIONS FROM STATIC AND MOBILE SENSORS}

In our mobile sensor assisted network, the coverage of a grid is achieved by the combined efforts of static and mobile sensors. A grid is said to be covered at time $t$ if either a static sensor in this grid is active or a mobile sensor resides in the grid at time $t$. To balance the workload, it is desirable to assign the static sensors with an identical activation probability $p$. An illustrative example of coverage is shown in Fig. 5 (refer to Fig. 1 for the distribution of the sensors in this example).

We now identify the necessary long-term coverage contributions from the two types of sensors. Clearly, for grid $i, i=0,1, \ldots, n^{2}-1$, the contribution from a mobile sensor depends on the fraction of time that the mobile sensor will be present in this grid; in other words, the probability that it travels to the grid. We denote this probability by $\pi_{i}$. The contribution from a static sensor in the grid is equal to 
its activation probability $p$ : the higher this probability, the better the coverage it provides.

We now focus on the optimal values of $p$ and $\pi=\left[\pi_{0}, \pi_{1}, \ldots, \pi_{n^{2}-1}\right]$. In the next section, we will present a concrete random walk model that achieves $\pi$.

To facilitate our discussion, we use $d(i)$ to represent the density of grid $i$, i.e., the number of static sensors in this grid. Let $M$ be the number of mobile sensors in the network. Given coverage requirement ratio $\delta$, the following formulation maximizes the network lifetime:

$$
\begin{gathered}
\text { minimize } \quad p \\
\text { s.t. } \quad \pi_{0}+\pi_{1}+\ldots+\pi_{n^{2}-1} \leq 1 \\
(1-p)^{d(0)} \times\left(1-\pi_{0}\right)^{M} \leq 1-\delta \\
(1-p)^{d(1)} \times\left(1-\pi_{1}\right)^{M} \leq 1-\delta \\
\vdots \\
(1-p)^{d\left(n^{2}-1\right)} \times\left(1-\pi_{n^{2}-1}\right)^{M} \leq 1-\delta
\end{gathered}
$$

where Eq. (3) gives the contribution constraint of each mobile sensor, and Eqs. (4) - (6) ensure the coverage ratio of the grids, i.e., if Eqs. (4) - (6) are satisfied, the overall expected coverage ratio is greater than $\delta$.

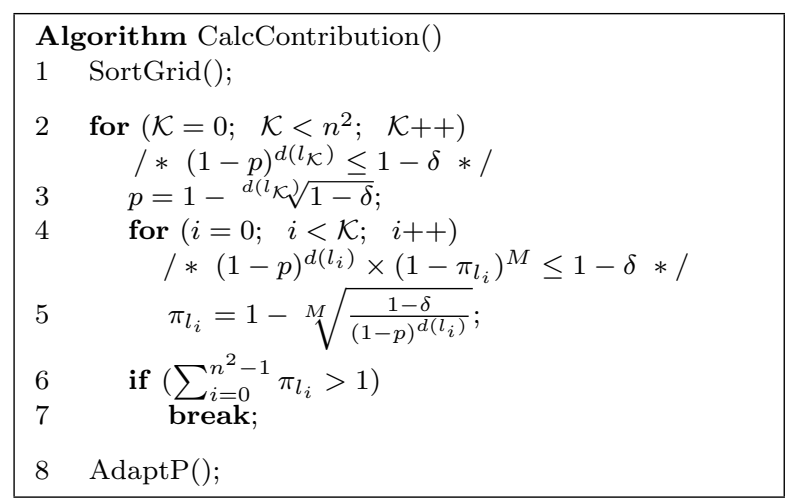

Fig. 6. Algorithm CalcContribution()

We present algorithm CalcContribution() that solves this optimization problem (see Fig. 6). The intuition behind this algorithm is that the network lifetime is determined by the sensors in the grid with the least number of static sensors as these sensors will have the highest activation probability and the shortest lifetime. We 


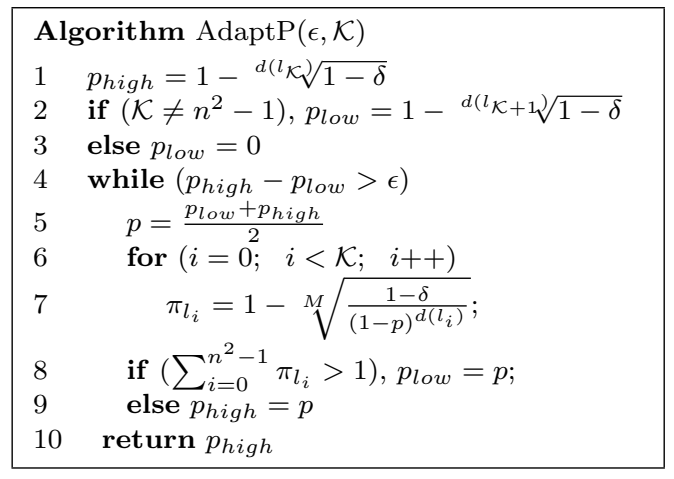

Fig. 7. Algorithm AdaptP()

thus first put all the mobile sensors to assist the grid with the least number of static sensors; and see whether the activation probability of the sensors in this grid can be reduced to the same activation probability of the grid with the second least number of static sensors. If we have enough mobile sensors (notice here the mobile sensor may not need to stay put in the grid, but only contribute a share of its presence in this grid), we continue to the grid with the third least number of static sensors. We stop until all the mobile sensors are fully exploited. In CalcContribution(), we first invoke subroutine SortGrid() to sort the grids in ascending order of their densities. Let $l_{i}$ represent the index of the grid with rank $i$ after sorting, i.e., $d\left(l_{0}\right) \leq d\left(l_{1}\right) \leq \ldots \leq d\left(l_{n^{2}-1}\right)$. We then search for $\mathcal{K}$, the rank after which the grids are dense enough to be covered by the static sensors only. We start searching for $\mathcal{K}$ from 0 , and evaluate the $p$ for the current setting of $\mathcal{K}$. If we can find a valid $p$ and $\pi_{l_{i}}$, then we increase $\mathcal{K}$, until $\sum_{i=0}^{n^{2}-1} \pi_{l_{i}}>1$ (intuitively, this says that the potential of the mobile sensors is fully exploited) or $\mathcal{K}$ reaches $n^{2}$. In this process, $p$ is decreasing because additional assistance from the mobile sensors is introduced after each iteration.

Note that $p$ is a real number but $\mathcal{K}$ is discrete. Hence, after the above process terminates, we in fact have an upper-bound on $p$ corresponding to $\mathcal{K}$, and a lowerbound on $p$ corresponding to $\mathcal{K}+1$. To find the optimal and practical $p$, we invoke a subroutine AdaptP() (in Fig. 7), which performs a binary search for the $p$ and adjusts $\pi_{l_{i}}$ accordingly. The termination of this subroutine depends on the precision of $p$, i.e., $\epsilon$, which is usually a predefined value. In our experiments, the depth of the binary search of the subroutine of $\operatorname{AdoptP}()$ is set to a constant factor of four.

The next theorem states that algorithm CalcContribution provides a solution that can be arbitrarily close to the optimal solution. Its running time is independent of the number of sensors.

THEOREM 1. Algorithm CalcContribution() provides a solution p that is at most $\epsilon$ from the optimal solution $p^{*}$ where $\epsilon$ can be arbitrarily small. Given a fixed $\epsilon$, the complexity of the algorithm is $\max \left(O\left(N^{2}\right), O(N \log \epsilon)\right)$ where $N$ is the number of grids. This is independent of the number of sensors.

Proof. We first prove the optimality. Assume that there is an optimal solution $p^{*}$. With this $p^{*}$, we can calculate the associated minimum $\pi_{l_{i}}^{*}$ that satisfy Eqs. 
(4) - (6). We will first show that with $p^{*}$ and $\pi^{*}$, the grids that are not covered by mobile sensors are the same as that found from algorithm CalcContribution().

Assume $\exists i, \pi_{l_{i}}^{*}=0$. We consider the grid $l_{i^{*}}$ with the minimum density among all these grids that are not covered by mobile sensors. This $i^{*}$ must be coincident with $\mathcal{K}$ found from CalcContribution(). Otherwise, since $p>p^{*}$, then $i^{*}>\mathcal{K}$. In this case, as $\sum_{i} \pi_{i} \leq 1$, the CalcContribution() will continue at line 6 and increase $\mathcal{K}$ until $\mathcal{K}=i^{*}$. If $\forall i, \pi_{l_{i}}^{*}>0, \mathcal{K}$ in line 6 will be increased until $\mathcal{K}=n^{2}-1$.

We then show that with subroutine $\operatorname{AdaptP}(), p$ must be within a distance of $\epsilon$ from $p^{*}$. This is straightforward as in subroutine $\operatorname{AdoptP}()$, the choice of $p_{\text {low }}$ always violates Eq. (3). Therefore, $p>p^{*}>p_{\text {low }}$. Since $p_{\text {high }}-p_{\text {low }}<\epsilon$ and $p=p_{\text {high }}$, then $p-p^{*}<\epsilon$.

The complexity of the algorithm is dominated either by line 2 and line 4 of the main algorithm, which is $N^{2}$, or line 4 and line 6 of the subroutine of $\operatorname{AdaptP}()$, which is $O(N \log \epsilon)$.

In practice, if there are many grids and $N$ is large, it may take a long time for a single mobile sensor to collect all the field information. In this case, we can first do a simple uniform partition of the field according to the number of mobile sensors and let each mobile sensor be responsible for the information collection in a subfield. As such, the initialization phase can be remarkably shortened.

For some applications, if there is an expected system lifetime $L$ to monitor the sensor field and a coverage quality requirement $\delta$, we can also compute the number of mobile sensors needed under current sensor deployment by a variant of CalcContribution(). The algorithm and discussion are in the Appendix of the paper.

\section{A RANDOM WALK MODEL FOR MOBILE SENSORS}

In the previous section, we obtained $\pi$, the long-term coverage contribution by the mobile sensors to the grids. It remains to show a concrete mobility model that can achieve this distribution. To this end, we demonstrate a viable and yet simple random walk model in this section. In this random walk model, the specific movement method for a mobile sensor when arriving at each different grid will be determined; and following this movement scheme, the long term probability $\pi$ that the mobile sensor will be presence in this grid will be guaranteed.

\subsection{A Random Walk Model}

In the random walk model, a mobile sensor will either stay in a grid, or move into an adjacent grid along four directions, ${ }^{4}$ as shown in Fig. 2. In this section, we will determine the probability for a mobile sensor to choose the five possible movements in the next time slot. We consider decisions depending only on the current grid where a mobile sensor resides. This results in a Markov chain where each grid is a state. We use $P_{i j}$ to denote the transition probability from grid $i$ to grid $j$. See Fig. 8 for an illustration. Given the long-run distribution $\pi$, this Markov chain obeys the following balance equations,

\footnotetext{
${ }^{4}$ For a mobile sensor in a boundary grid, it might have 3 or 2 directions to move only.
} 


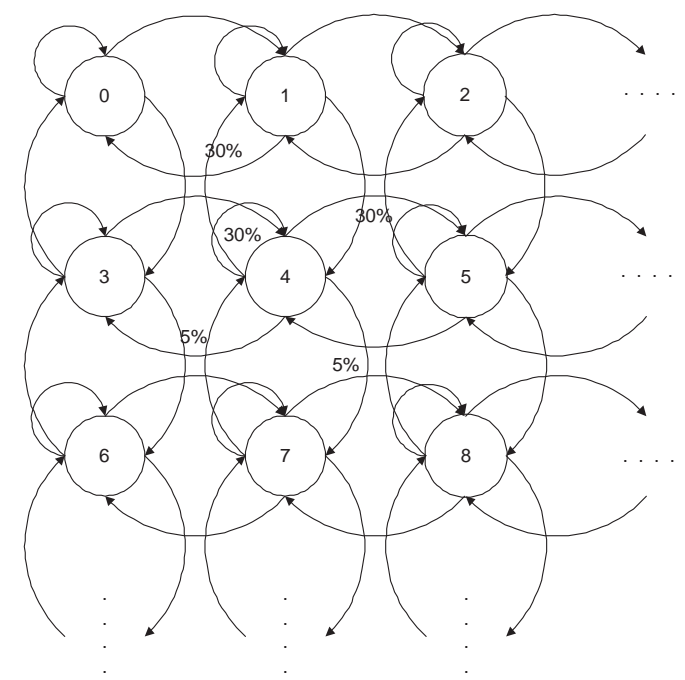

Fig. 8. Markov chain for the random walk model.

$$
\begin{gathered}
\pi_{j}=\sum_{k=0}^{n^{2}-1} \pi_{k} P_{k j}, \quad j=0,1, \ldots, n^{2}-1 \\
\sum_{k=0}^{n^{2}-1} \pi_{k}=1 \\
\sum_{j=0}^{n^{2}-1} P_{k j}=1, \quad \forall k \in\left[0, n^{2}-1\right] \\
0 \leq P_{i j} \leq 1, \quad \forall i, j
\end{gathered}
$$

$$
P_{i j}=0, \quad \forall i, j, \text { grids } i, j \text { not adjacent }
$$

where the first four equations are standard steady-state constraints for Markov chains [Karlin and Taylor 1998], and Eq. (11) suggests that no transition is possible for two non-adjacent grids.

Our problem now is to determine the transition probabilities $P_{i j}$ in this system of equations to reach the stationary distribution $\pi$. This is the inverse of the traditional "given transition probability, find stationary distribution" problem in a Markov chain.

First of all, we need to ensure that the $P_{i j}$ obtained can guarantee a limiting distribution $\pi$. By ergodic theorem [Ross 1989], a Markov chain that is aperiodic, irreducible and positive recurrent has a limiting distribution ${ }^{5}$. Since there are only

\footnotetext{
${ }^{5}$ Aperiodic means that $P_{i i}>0$. Irreducible means that all states are reachable from all other
} ACM Transactions on Computational Logic, Vol. V, No. N, October 2010. 
a finite number of states in our system, if our Markov chain is irreducible, it is positive recurrent. As such, if we ensure that the Markov chain is aperiodic and irreducible, it is sufficient to guarantee this $\pi$ exists. For ease of discussion, we now assume that $\pi_{k}>0$ for $k=0,1, \ldots, n^{2}-1$. We will generalize the solution later.

To ensure aperiodicity, we can set all the $P_{i i}$ to be strictly positive. To ensure irreducibility, the mobile sensors cannot be trapped in a grid or a group of grids; hence, we have an additional set of constraints:

$$
\forall i, \quad 0<P_{i i}<1,
$$

which indicates that whenever a mobile sensor moves into a grid, the probability that it will stay in this grid should be strictly less than 1. A stronger condition is

$$
P_{i j}>0, \forall i, j, \text { grids } i, j \text { are adjacent, }
$$

which ensures that the mobile sensor always has chance to move into a neighboring grid. Eq. (10) can then be replaced by

$$
0<P_{i j}<1, \quad \forall i, j \text { that are adjacent }
$$

It is not difficult to see that the above set of equations have multiple solutions. We now illustrate one solution set. Our strategy is to first find a set of solution to Eq. (7) and Eq. (8) and then try to satisfy all others. Notice that if $\pi_{k} P_{k j}=\pi_{j} P_{j k}$, Eq. (7) can be satisfied. We set $P_{k j}=\pi_{j}$ and $P_{j k}=\pi_{k}$ for all $P_{j k} \neq 0$ and $P_{k j} \neq 0$. This can always be achieved because either $P_{k j}$ and $P_{j k}$ are both strictly positive, or $P_{k j}=P_{j k}=0$. We then set $P_{i i}=1-\sum_{j=0}^{n^{2}-1} P_{i j}$, and it is easy to verify that $P_{i i}>0$. Therefore, Eqs. (7), (8) and (9), (11) are satisfied. Since $\pi_{k}, \pi_{j} \neq 0,1$ we have $P_{j k}, P_{k j} \neq 0,1$, and Eqs. (12), (14) are satisfied.

In summary, the solution set is

$$
\begin{aligned}
& P_{j k}=\left\{\begin{array}{l}
\pi_{k} \forall j \neq k \text { and } j, k \text { are adjacent; } \\
0 \quad \forall j \neq k \text { and } j, k \text { are not adjacent; }
\end{array}\right. \\
& P_{j j}=1-\sum_{k=0}^{n^{2}-1} P_{j k} \quad \forall j \neq k
\end{aligned}
$$

Here we emphasize again that we assume $\pi_{k}>0$ for $k=0,1, \ldots, n^{2}-1$. In section 5.3 , we will investigate an interesting impact of $\pi_{k}=0$, i.e., that certain grids do not need assistance from the mobile sensors. As the transition probability $P_{i j}$ for each grid is calculated from $\pi_{i}$, it is guaranteed that given all the mobile sensors follow $P_{i j}$, the long term contribution will be achieved.

\subsection{Boosting Movement}

It is worth noting that the definition of coverage quality (Definition 1 in Section 3.2 ) does not account for the moving frequency of the mobile sensors, nor the

states. Positive recurrent means that the sensor will return to a state within finite time. 


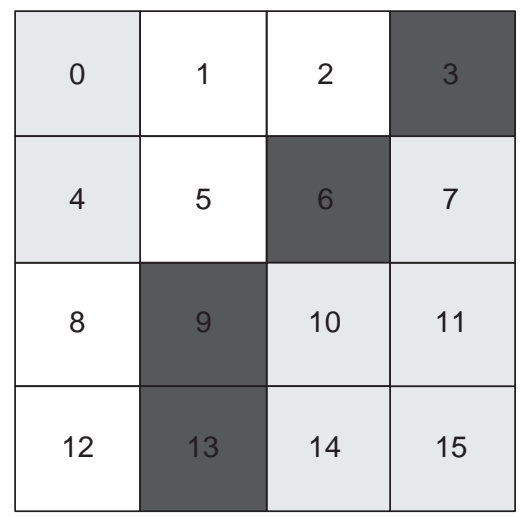

Fig. 9. Wall effect. Darker grids have denser static sensors.

convergence time of the system. A lazy movement, where there is a high probability for the mobile sensors to stay in the same grid, would achieve the same coverage ratio. An extreme example is one-time repositioning of the mobile sensors: a higher fraction of the sensor field can be covered, but the coverage could still be unbalanced or even with holes if the number of mobile sensors is not enough.

Our random walk model can effectively solve this problem by adaptively setting the transition probabilities, allowing a wide range of movement frequencies. The strategy is to adjust the existing solution within the constraints to obtain another viable solution set. Specifically, to satisfy Eq. (7), we only need to have $\pi_{k} P_{k j}=$ $\pi_{j} P_{j k}$; thus setting $P_{k j}=\alpha \pi_{j}$ and $P_{j k}=\alpha \pi_{k}$ also works given $\alpha>0$. Let $\alpha_{l}, \alpha_{u}, \alpha_{r}, \alpha_{d}$ denote the adjustment factors for the four directions. To achieve a higher moving frequency, we can increase $\alpha_{l}, \alpha_{u}, \alpha_{r}, \alpha_{d}$, and the constraints will still be satisfied as long as the sum of the outgoing probabilities in a grid is less than 1. In our experiments, we set a threshold for $P_{i i}$ : if a $P_{i i}$ is greater than the threshold, we increase the $\alpha$ 's until all $P_{i i}$ 's are less than the threshold, or there is no possible further reduction. We call the scheme after this adjustment aggressive movement.

\subsection{The Wall Effect and Solutions}

We have assumed that $\pi_{i}$ is non-zero in the previous Markov chain calculation. In practice, $\pi_{i}$ can be zero for dense grids, i.e., those ranked higher than $\mathcal{K}$ in algorithm CalcContribution(). These grids will not get assistance from the mobile sensors and can simply be ignored in forming the Markov chain, if they are sparsely distributed. However, if a collection of such grids are connected, a wall can be formed, which partitions the field into two or more disjoint subfields. Given the presence of a wall (or multiple walls), a mobile sensor can not move freely in the whole field, and the expected distribution is no longer achievable. An example of this wall effect is shown in Fig. 9 where grids 3, 6, 9, 13 have dense static sensors and thus form a wall, splitting the fields into two subfields. Grid 0 and 4 also have dense static sensors. Compared to the wall grids, they still need some assist from mobile sensors. We call them semi-walls as these grids make traveling in subfield $(0,1,2,4,5,8$, 
12) difficult, i.e., it may take a long time for the mobile sensors in grids $1,2,5$ to reach grid 8,12 . As such, the coverage of the non-wall grids strongly depends on the initial placement of the mobile sensors, and a strategic allocation of the mobile sensors to the subfields is thus necessary.

5.3.1 Mobile Sensor Allocation for Subfields. Assume that, after invoking algorithm CalcContribution in the initial stage, the sensor field is divided into $C$ subfields by walls. It is easy to see that the number of mobile sensors needed in each sub-field (excluding the wall grids) is independent of other subfields. We thus focus on a particular subfield, e.g., the $k$ th one. Assume this subfield includes $C^{k}$ grids, and similar to the notations used previously, let grid $l_{i}^{k}$ be the $i$ th rank in this subfield after sorting in ascending order of the densities, i.e., $d\left(l_{0}^{k}\right) \leq d\left(l_{1}^{k}\right) \leq \ldots \leq d\left(l_{C^{k}-1}^{k}\right)$. Let $M^{k}$ be the number of mobile sensors to be assigned to this subfield. Our objective is to find the minimum $M^{k}$ that provides the desired coverage for this subfield. This problem can be formulated as follows:

$$
\begin{gathered}
\text { minimize } M^{k} \\
\text { s.t. } \quad \pi_{l_{0}^{k}}+\pi_{l_{1}^{k}}+\ldots+\pi_{l_{C^{k}-1}^{k}} \leq 1 \\
\left(1-p_{\text {min }}\right)^{d\left(l_{0}^{k}\right)} \times\left(1-\pi_{l_{0}^{k}}\right)^{M^{k}} \leq 1-\delta \\
\left(1-p_{\min }\right)^{d\left(l_{1}^{k}\right)} \times\left(1-\pi_{l_{1}^{k}}\right)^{M^{k}} \leq 1-\delta \\
\vdots \\
\left(1-p_{\text {min }}\right)^{d\left(l_{C^{k}-1}^{k}\right)} \times\left(1-\pi_{l_{C^{k}-1}^{k}}\right)^{M^{k}} \leq 1-\delta
\end{gathered}
$$

where $p_{\min }$ is the optimal value of $p$ obtained in CalcContribution. To maximize the expected network lifetime, this value should still be identical for all the static sensors, even in the presence of subfields.

We can iteratively reduce $M^{k}$ starting from $M-\sum_{j=0}^{k-1} M^{j}$. We allocate mobile sensors to each subfield one by one and, for the $k$ th subfield, we start with the remaining mobile sensors after assigning all $k-1$ subfields. We then calculate the the corresponding $\pi_{l_{i}^{k}}$ in each iteration. We stop until Eq. (17) is violated, (intuitively, this means that fewer sensors cannot provide necessary coverage). We thus obtain optimal $M^{k}$ and $\pi_{l_{i}^{k}}$. Since the grids within the subfield all have $\pi_{l_{i}^{k}}>0$, we can set the transition probabilities as before. The transition probabilities also guarantee that a mobile sensor will remain in its subfield during the random walk.

It is worth noting that after we calculate each $M^{k}$ individually, it is possible that $\sum_{k=0}^{C} M^{k}>M$. This is because a sensor cannot be allocated fractionally. Given this negative impact of the walls, we need to increase $p_{\min }$ by decreasing $\mathcal{K}$; the contribution from the static sensors is thus increased. We continue until a $\mathcal{K}$ is found such that $\sum_{k=0}^{C} M^{k} \leq M$. 
5.3.2 Subfield Partitioning. Besides the wall grids, other dense grids may have a very small $\pi_{i}$, implying that the mobile sensors should seldom visit them. Two examples are the grids 0 and 4 in Fig. 9. These two grids make a smooth walking in subfield $(0,1,2,4,5,8,12)$ difficult and will significantly increase the convergence time of the system.

In the presence of semi-walls, we can further partition the subfields to balance the movement of the mobile sensors. Again, since the mobile sensors cannot be allocated fractionally, we have to strike a balance between the coverage and convergency. In our experiment, we set a threshold for the grids of semi-walls and show that the convergence time improves noticeably.

\section{SENSOR COLLABORATIONS}

So far we have established the respective contributions from static and mobile sensors, and the activation and movement strategies for them. This framework is easy to implement as it involves node interactions in the initial period only, and all the remaining operations are randomly and independently performed in a distributed fashion. Within this basic framework, various node interactions/collaborations could be introduced to further enhance the system performance. More importantly, though the framework statistically guarantees the coverage, the independent behavior will result in overlapping coverage by the mobile and/or static sensors. That is, if there is no knowledge exchange between neighboring sensors, multiple sensors may cover the grid simultaneously; which is a waste. We thus outline a simple yet effective node collaboration scheme.

The key idea here is to prevent overlapping coverage of a grid by different sensors. Intuitively, if a static sensor finds that there is a mobile sensor or another static sensor in work, it can return to sleep mode. Or if two mobile sensors find that they are trying to enter the same grid at the same time slots, one should restrain. To this end, we introduce a sensor collaboration protocol with two contention phases (The pseudo-code for the collaboration scheme can be found in Fig. 10.). Without loss of generality, we consider a time slot starting at $T_{i}$ of length $T$. The first phase $\left[T_{i}-t, T_{i}\right]$ is used for contention between mobile sensors to enter one certain grid; the second phase $\left[T_{i}, T_{i}+t\right]$ is used for suppressing multiple activation of the static sensors. Here, $t$ is a fixed parameter such that $t \ll T$.

In $\left[T_{i}-t, T_{i}\right]$, mobile sensor $m_{j}$ first decides which grid it will enter in the next time slot. Then, $m_{j}$ randomly generates a number $t_{j} \in[0, t]$ and, at time $T_{i}-t_{j}$, sends a probe message to the sensors in the selected grid. If the grid has a mobile sensor or an active static sensor, it will allow $m_{j}$ to enter in the next slot only if $m_{j}$ is the first one sending the probe message. In $\left[T_{i}, T_{i}+t\right]$, each static sensor also generates $t_{j} \in[0, t]$, and, at time $T_{i}+t_{j}$, activates itself with probability $p$ and broadcasts a probe message to its neighbors in the same grid. If a neighbor is a mobile or an already activated static sensor, it will reply by a reject message; The newly activated sensor thus has to deactivate itself to save energy.

We see that basically, the mobile sensors will randomly select a time $t_{j}$ in the window of $\left[T_{i}-t, T_{i}\right]$ and mobile sensor who has the earliest $t_{j}$ will enter and cover the grid in the next time slot. This mobile sensor will also suppress the activation of all the static sensors. If there is no mobile sensor, the static sensors will randomly 


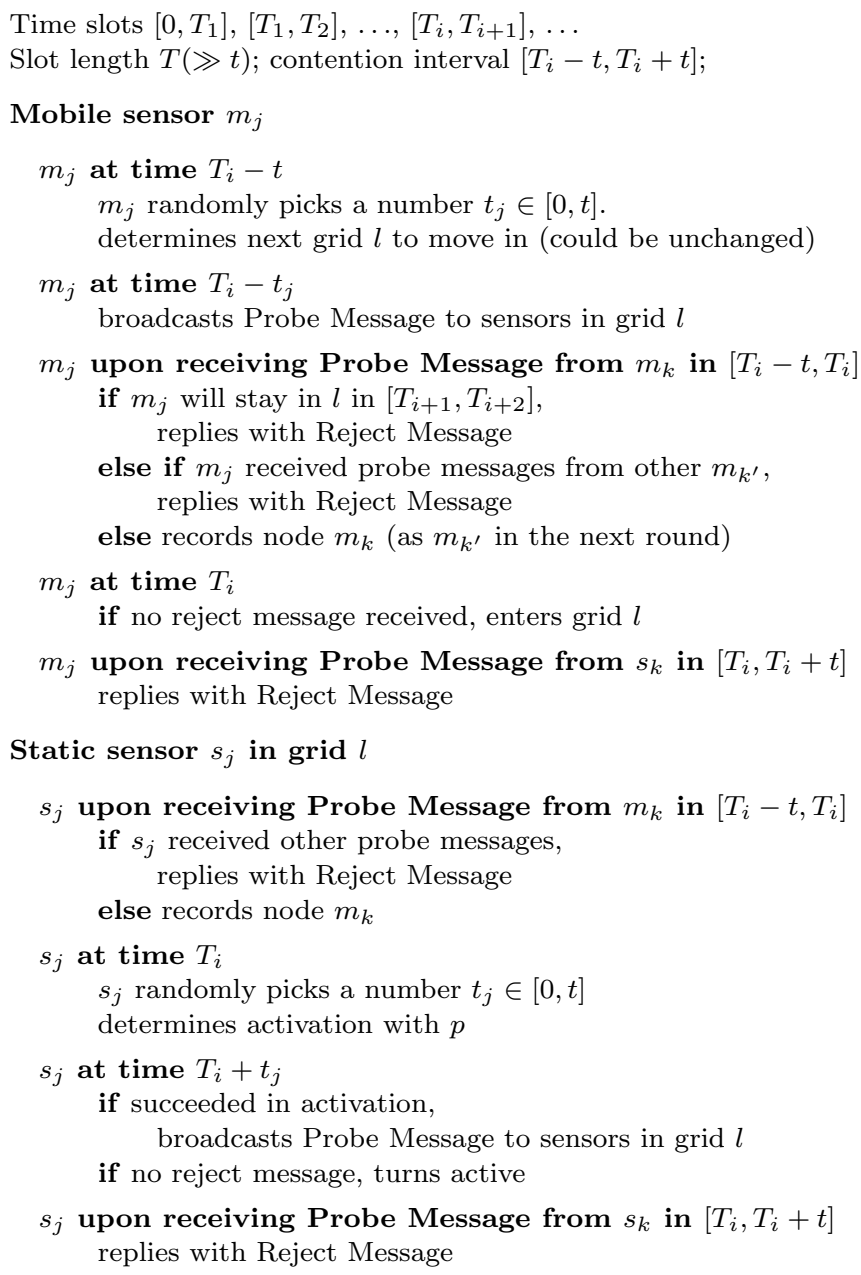

Fig. 10. Sensor Collaboration Protocol.

select a time in the window of $\left[T_{i}, T_{i}+t\right]$; and the sensor who has the earliest time will become active to cover this grid for the next the time slot. Thus, we have the following observation.

OBSERVATION 1. There will be at most one sensor that is active in each grid during any time slot.

The overhead of this protocol depends on the number of sensors who activated simultaneously. We will show the overhead in our simulation.

\section{PERFORMANCE EVALUATION}

In this section, we evaluate the performance of the mobile sensor assisted network in field coverage through simulations. We focus on the following typical measures: coverage quality, network lifetime, and convergence time. 


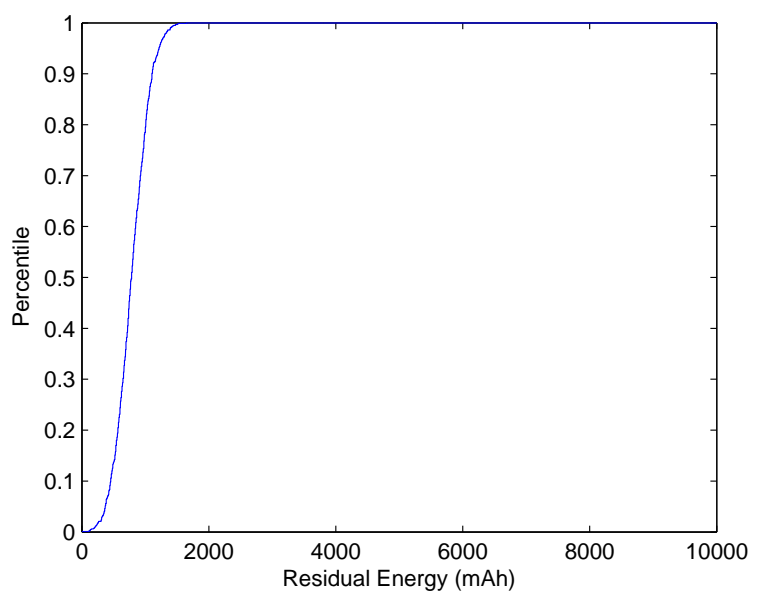

Fig. 11. Residual energy after the death of the first sensor.

In our simulation, we deploy 1000 static sensors in a field of $140 \mathrm{~m} \times 140 \mathrm{~m}$ and the sensor field is partitioned into 100 virtual grids. The battery power for each sensor is $10000 \mathrm{mAh}$, and can last for one day with persistent activation. We neglect the energy cost during dormant states.

We have examined the energy consumption status of the static sensors in our system. Fig. 11 shows the cumulative distribution curve of the residual energy after the death of the first sensor. We can see that at this time more than $70 \%$ of the sensors has residual energy less than $1000 \mathrm{mAh}(10 \%$ of the total energy reserve). It implies that the remaining operation time of the system is very limited, and the lifetime of the first dead sensor thus serves as a legible measure for the system lifetime.

We compare our mobile assisted scheme with both static sensor only scheme and a state-of-the-art one-time reposition scheme [Wang et al. 2003]. We applied its bidding protocol so that the mobile sensors will move to the positions where there are less number of static sensors. As this one-time reposition scheme requests all the sensors to stay awake after reposition, we modified it so that the sensors will switch between sleep and awake states.

Unless otherwise specified, the following default parameters are used in our simulation: The expected coverage quality is $\delta=0.85$, and the length of each time slot is 1 minutes. The total number of time slots is set to 1000. Each point in our figures is the average of 100 independent experiments.

\subsection{Contribution of Mobile Sensors}

In first set of experiments, we deployed different number of mobile sensors in the field to observe their effectiveness. In Fig. 12, we show the network lifetime as a function of the number of mobile sensors. The number of mobile sensors varies from 20 to 60 , which accounts for only a small portion of all the sensors. For comparison, we also plot the results with static sensors only; to ensure fairness, in this case, we deployed additional static sensors (the same amount as mobile sensors), which are 


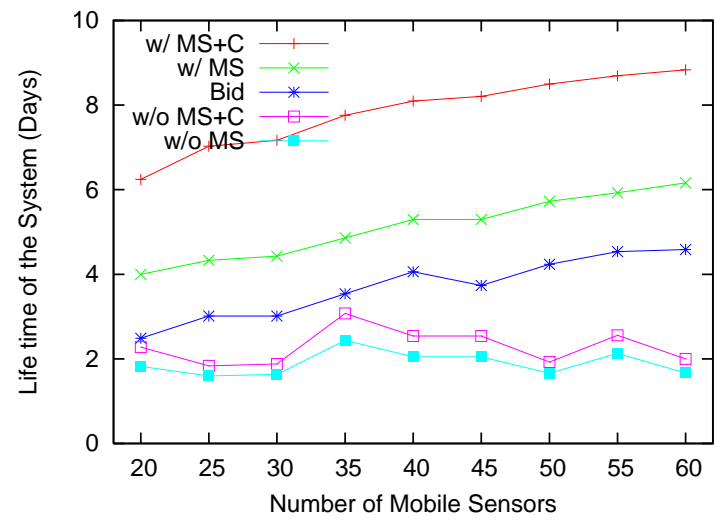

Fig. 12. Comparison of the system lifetime with and without mobile sensors, and with and without collaborations.

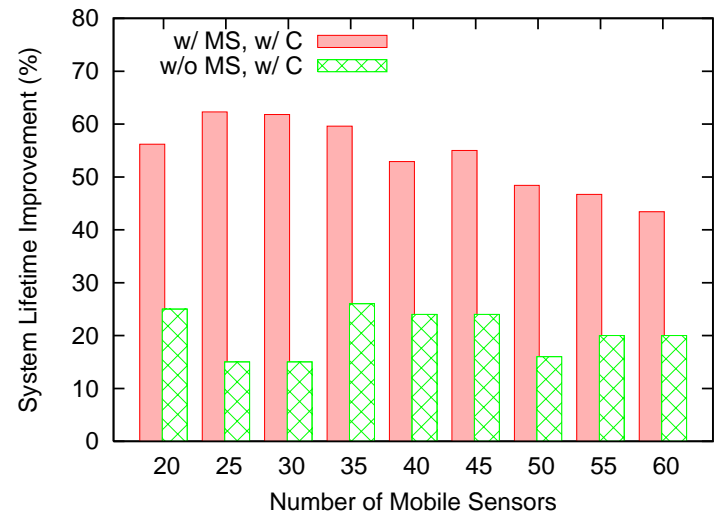

Fig. 13. System lifetime with or without collaborations.

equipped with extra-batteries to remain active throughout the experiments. In our figures, we use w/ MS, w/o MS to denote the experiments with or without mobile sensors; w/ C, w/o C to denote the experiments with or without using the sensor collaboration protocol; and Bid to denote the one-time reposition scheme [Wang et al. 2003].

We observe that the use of mobile sensors substantially increases the network lifetime. For example, consider the case where there are 50 mobile sensors, the lifetime (w/ MS, w/o C) is three times longer than without mobile sensors (w/o MS, w/o C). In addition, we see that the lifetime improves steadily when more mobile sensors are deployed. On the contrary, by adding a few static sensors only, there is no clear improvement of the system lifetime. The performance of one-time reposition (Bid) is also better than without mobile sensors. The performance increases as the number of mobile sensors increases. Nevertheless, the performance is much worse than our mobile assisted scheme, especially when collaboration is used. Node collaboration also improves the lifetime for both with and without mobile sensors, 


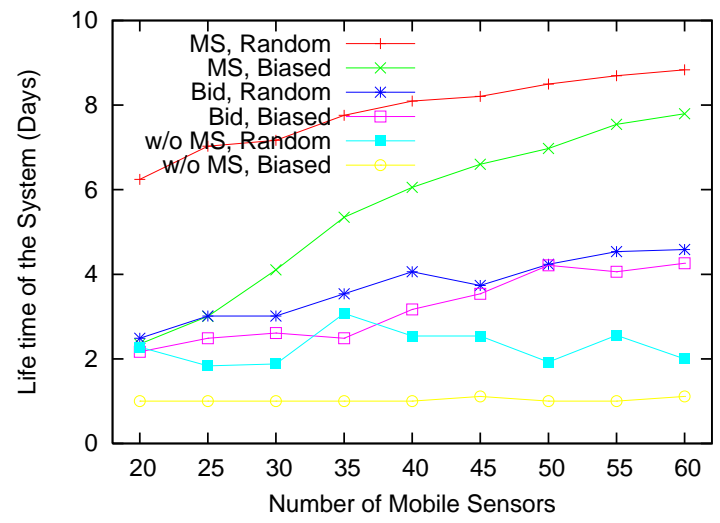

Fig. 14. Comparison of the system lifetime for uniform and biased distributions of static sensors.

but more substantially if mobile sensors are used. The improvement percentage is plotted in Fig. 13. We can see that without mobile sensor (w/o MS, w/ C), there is a $10 \%$ to $20 \%$ lifetime improvement with sensor collaboration compared to without collaboration. If mobile sensors are used, this effect is much pronounced. This is because without mobile sensors, the lifetime is constrained by the grids with fewer sensors, resulting in smaller chance of suppressing redundant activations. Since node collaboration substantially improves the system performance, for the rest of our experiments, we will focus on the performance of the system with collaboration only.

We next consider the effect of two different distributions of the static sensors. First, we deployed the static sensors randomly and uniformly. Second, we added some bias on the distribution, where the right side of the sensor field was two times denser than the left side of the sensor field. Fig. 14 shows the comparison results. Not surprisingly, the lifetime has reduced in biased distribution since the system is more stressed. With assistance from mobile sensors, however, the situation improves fast; for example, with 20 mobile sensors, the lifetime is only marginally better than with no mobile sensors at all, whereas with 60 mobile sensors, the lifetime is less significantly affected by the bias of the distribution. We see this effect both in our mobile assisted scheme and the one-time reposition scheme; though the one-time reposition scheme has worse performance. This clearly shows the inherent adjustment capability of using mobile sensors.

\subsection{Convergence Time}

We now study some internal parameters of our mobile assisted scheme. We consider the convergence time of the network, in particular, the effect of moving speed of the mobile sensors. We simulated 50 mobile sensors and 1000 static sensors in the sensor field. In initialization, the whole sensor field was partitioned into subfields by walls. All mobile sensors belonging to the same subfield were dispatched to the grid with the highest index in this subfield. Fig. 15 shows the coverage quality over time for both aggressive and lazy movements. We see that if there are high transition probabilities between adjacent grids, the convergence time is much smaller. For 


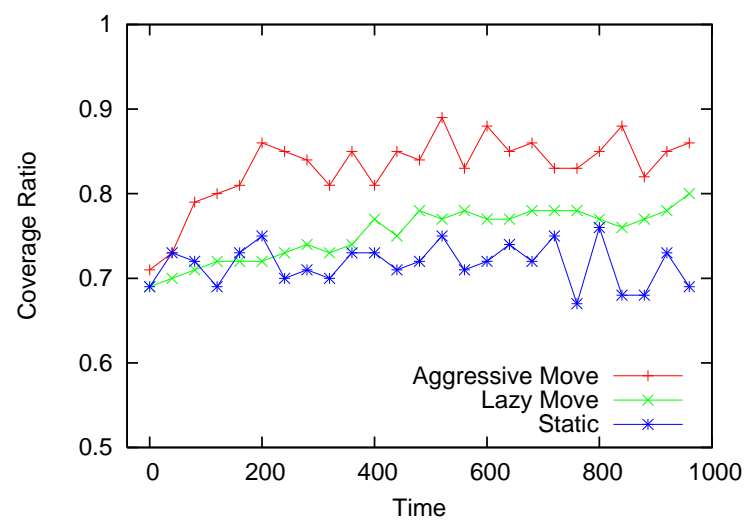

Fig. 15. Comparison of the coverage ratio as a function of running time for varying movement patterns.

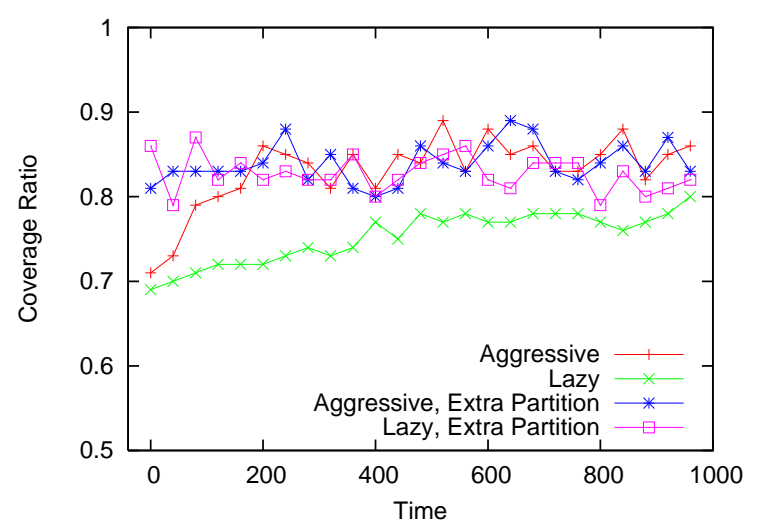

Fig. 16. Comparison of the coverage ratio as functions of running time with partitioning.

example, with aggressive movement, the system reaches $85 \%$ coverage after 200 minutes, while lazy movement has yet to reach this ratio after 1000 minutes. We can also see from Fig. 15, that the coverage ratio with static sensors only is only around $70 \% .^{6}$

We consider the effect of finer partitioning of the subfields. From Fig. 16, we see that finer partition improves the convergence time with both aggressive and lazy movements.

These experiments clearly show that the walls and semi-walls in the field would remarkably affect the convergence of the system, and our allocation algorithms for the mobile sensors can effectively solve this problem.

\footnotetext{
${ }^{6}$ Note that the curves go up and down in the figure. This is because each point in the figure represents one specific time. Our algorithms target on a statistical coverage; and at each point of time, we observe variations in the coverage ratio.
}

ACM Transactions on Computational Logic, Vol. V, No. N, October 2010. 


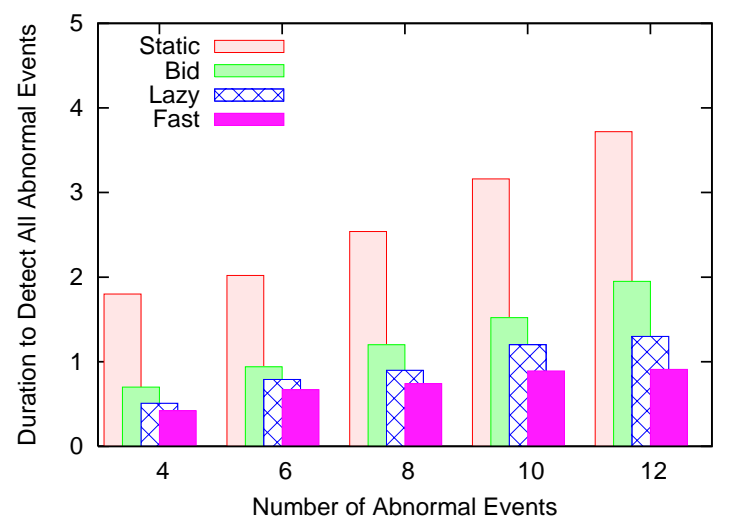

Fig. 17. Duration (minutes) to detect all abnormal events.

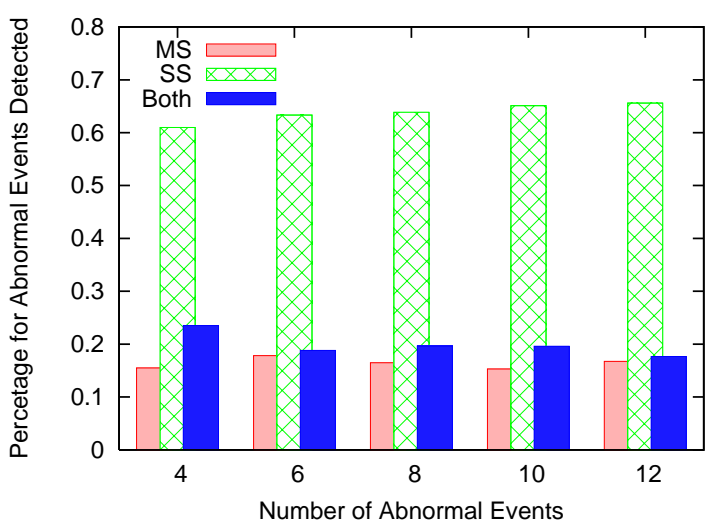

(a)

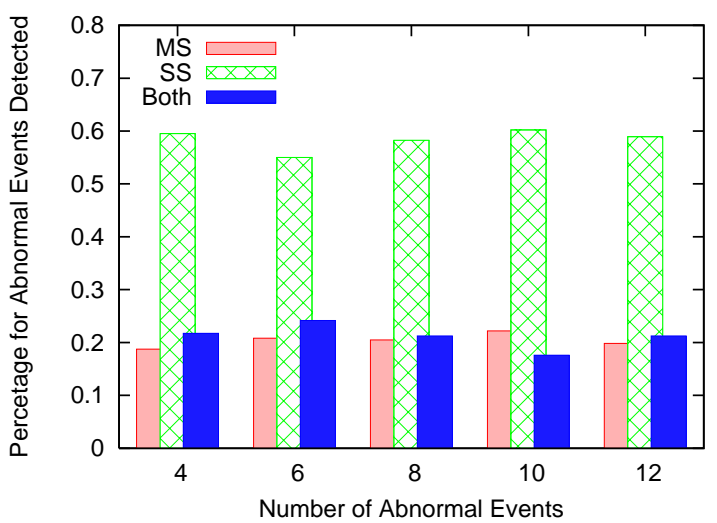

(b)

Fig. 18. Abnormal event detection. SS: Detected by static sensors only; MS: Detected by mobile sensors only; Both: Detected by both. (a) Mobile sensors with lazy movement. (b) Mobile sensors with aggressive movement.

ACM Transactions on Computational Logic, Vol. V, No. N, October 2010. 


\subsection{Aggressive Movement in Event Detection}

While finer partitioning makes the the convergence time of lazy movement close to that of aggressive movement, we argue that aggressive movement can be much more effective than lazy movement in abnormal event detection.

We randomly generated abnormal events in the sensor field. In Fig. 17, we show the time needed to detect all these events for three strategies, namely, aggressive movement, lazy movement and without mobile sensors. Not surprisingly, the more abnormal events there are, the longer it takes to find all of them. We see that with aggressive movement, the detection time is not only shorter than the other two, but also increases more slowly when the number of abnormal events increases. The gain obtained from aggressive movement compared to lazy movement is around $5 \%$ to $15 \%$. Notice that this is achieved neither by increasing the number of the mobile sensors nor by increasing their physical speeds, but simply by improving the transition probabilities between the grids. We see that the one-time reposition scheme is only slightly slower than in abnormal event detection. We should recall that the one-time reposition scheme achieves this by significantly shorter system lifetime. Finally, note that the detection time of using static sensors only is remarkably longer than the other three. In fact, in some tests, the events can never be fully detected if the grids has no any static sensor; we set an expiration time of 20 in such cases, which explains the high average detection time.

To further understand the contributions from static and mobile sensors, we show in Fig. 18 the ratio of the abnormal events detected by different types of sensors, namely, static, mobile, or both. We see that the static sensors are still the main source in coverage, detecting $55 \%$ to $60 \%$ of the abnormal events alone. The mobile sensors detect around $20 \%$ and for the other $20 \%$ cases, static and mobile sensors observe the abnormal events simultaneously. Again, this shows that a small number of mobile sensors can serve as an effective method for field coverage. Fig. 18 (a) and (b) demonstrate the scenario where the mobile sensors adopt lazy movement and aggressive movement strategies. We can also see that, if aggressive movement is adopted, the mobile sensors become more effective in detecting abnormal events.

The overhead of different sensors in our collaboration protocol is illustrated in Fig. 19. Though the total time slots is 1000 in our simulation, here the overhead is only averaged over the total time slots that the sensor is in active. We see that the overhead is around 7 packets per time slot. Our protocol is localized, resulting a moderate overhead.

\section{GENERALIZING GRID STRUCTURE}

We have assumed a square grid structure for the field in our study, which has also been widely adopted in this research area. A limitation of the square grid structure is its inflexible moving directions. As an example, consider an abnormal event is $R_{m}+d$ away from a mobile sensor, where $R_{m}$ is the sensing range of a mobile sensor and $d$ is a small distance. If the event happens in the upper-right direction (see Fig. 20 (a)), then because the two grids are not adjacent, the mobile sensor will detect it after moving at least twice. A hexagon structure, like that in the cellular network, will perform better in this case. As shown in Fig. 20 (b), only if the abnormal event is more than $2 R_{m}$ away from the mobile sensor can it avoid 


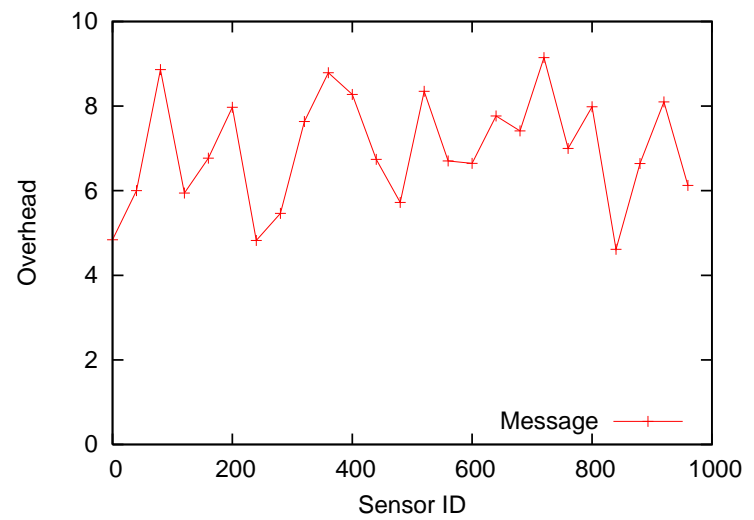

Fig. 19. Overhead of the collaboration protocol.

being possibly detected in the next sensor movement.

Our mobile sensor assisted architecture and the mobility models are not restricted to the square grid structure. They can easily accommodate the hexagon or even more general polygon structures. Intrinsically, the field is divided into subregions; and our algorithm specifies that the mobile sensors needs to move to those subregions that have less number of static sensors. Whether the subregion is a grid or not does not affect the algorithm. In fact, algorithm CalcContribution does not depend on the grid structure. The next state of Markov chain for the mobile sensors depends on the neighbors of each subregion. Therefore, some transitions need to be added in the Markov chain, e.g., for the hexagon structure, six transitions are needed against the four for the square grid case, and other calculations remain unchanged.

\section{CONCLUSIONS AND FUTURE WORK}

In this paper, we proposed a mobile sensor assisted network architecture, which is cost-effective and combines the advantages of both static and mobile sensors for field coverage. We offered an optimal algorithm for calculating the coverage contributions, which fully explores the potentials of the mobile sensors and maximize the network lifetime. We further presented a random walk model for the mobile sensors. The model is low-overhead and fully distributed. Its parameters can be fine-tuned for the same long-term coverage contribution and yet with different moving frequencies. As such, our model is general enough to match the moving capability of various mobile sensors and the demands from a broad spectrum of applications. We also showed the wall effect in this architecture, and developed an effective field partitioning and mobile sensor allocation algorithm. Under our basic framework, we developed in-network collaboration which further improves system performance.

\section{APPENDIX}

Given the expected system lifetime $L$ and the coverage quality requirement $\delta$, the number of mobile sensors needed can be calculated by Algorithm CalMobile() in Fig. 21. In this algorithm, we first translated the the expected system lifetime $L$ into 

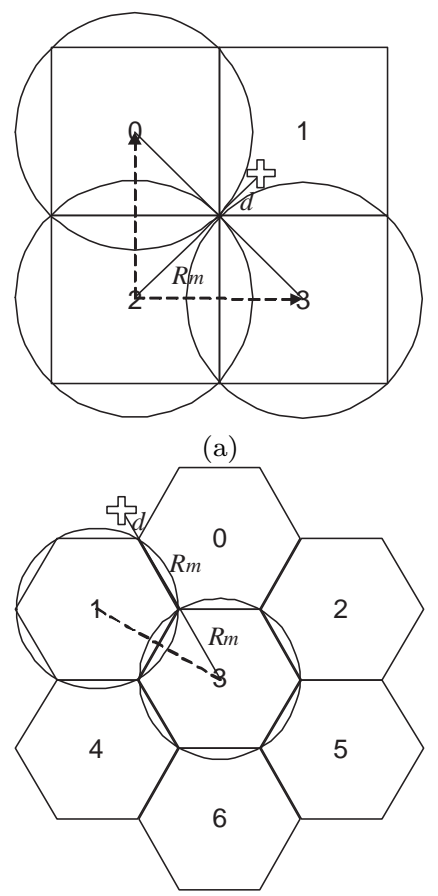

(b)

Fig. 20. Abnormal event is shown as a cross. (a) Square grid structure. The mobile sensor in grid 2 has to move at least two steps to detect the abnormal event, which is only slightly more than $R_{m}$ far away from the mobile sensor. (b) Hexagon structure. Only if the abnormal event is greater than $2 R_{m}$ away from the mobile sensor, can it avoid being possibly detected in next sensor movement.

the activation probability $p$ in line 2. We increase $M$ until Eq. 3 is satisfied. This algorithm can be performed at the Initialization phase after the sensor deployment. More mobile sensors is then added if necessary.

The running time of this algorithm is upper bounded by the situation that there is no static sensors in the field at all. Thus, by setting $p=0$ to line 6 , the termination condition in line 9 is $(1-\sqrt[M]{1-\delta}) \times N \leq 1$. Recall that $N$ is the number of grids in the field. Therefore, the upper bound is $M=\log _{1-\frac{1}{N}}(1-\delta)$. To show the basic idea and simplify CalMobile(), we take a linear implementation in searching for $M$. The running time of CalMobile() is thus $\log _{1-\frac{1}{N}}(1-\delta)$. A binary search of $M$ can be easily applied and the running time can be reduced with a logarithmic factor.

\section{ACKNOWLEDGMENT}

D. Wang's work was supported by grants Hong Kong PolyU/G-YG78, A-PB0R, APJ19, 1-ZV5W, and RGC/GRF PolyU 5305/08E. J. Liu's work was supported by a Canada NSERC Discovery Grant, an NSERC DAS Grant, an NSERC Strategic Project Grant, and a MITACS Project Grant. Q. Zhang's work was supported in part by Hong Kong ITF ITP/023/08LP and National Natural Science Foundation of China with grant no. as 60933012. 


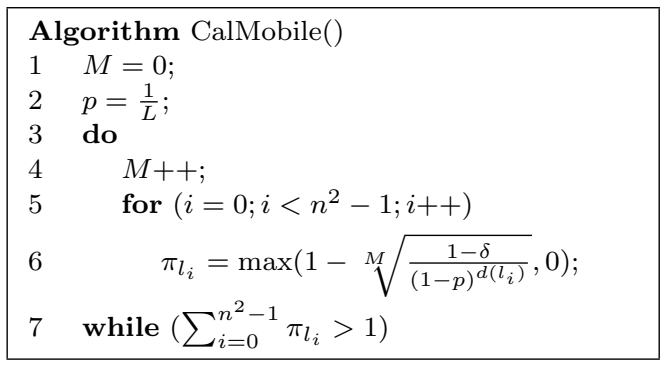

Fig. 21. Algorithm CalMobile()

\section{REFERENCES}

Akyildiz, I., Su, W., Sankarasubramaniam, Y., and Cayirci, E. 2002. A survey on sensor networks. IEEE Communications Magazine 40, 8 (Aug.), 102-114.

Albowitz, J., Chen, A., , And Zhang, L. 2001. Recursive position estimation in sensor networks. In Proc. IEEE ICNP'01. Riverside, CA.

Bisnik, N., Abouzeid, A., ANd Isler, V. 2006. Stochastic event capture using mobile sensors subject to a quality metric. In Proc. ACM MOBICOM'06. Los Angeles, CA.

Bulusu, N., Heidemann, J., And Estrin, D. 2000. Gps-less low cost outdoor localization for very small devices. IEEE Personal Communications Magazine 7, 5 (Oct.), 28-34.

Chang, J. and Tassiulas, L. 2000. Energy conserving routing in wireless ad-hoc networks. In Proc. IEEE INFOCOM'O0. Tel-Aviv, Israel.

Gao, J., Guibas, L., Hershburger, J., Zhang, L., and Zhu, A. 2001. Geometric spanner for routing in mobile networks. In Proc. ACM MOBIHOC'01. Long Beach, CA.

Gui, C. and Mohapatra, P. 2004. Power conservation and quality of surveillance in target tracking sensor networks. In Proc. ACM MOBICOM'04. Philadelphia, PA.

Heinzelman, W., Chandrakasan, A., and Balakrishnan, H. 2000. Energy-efficient communication protocol for wireless microsensor networks. In Proc. Hawaaian International Conference on Systems Science (HICSS'00). Wailea Maui, HI.

Howard, A., Mataric, M., and Sukhatme, G. 2002a. An incremental self-deployment algorithm for mobile sensor networks. Autonomous Robots Special Issue on Intelligent Embedded Systems 13, 2, 113-126.

Howard, A., Mataric, M., and Sukhatme, G. 2002b. Mobile sensor network deployment using potential field: a distributed scalable solution to the area coverage problem. In Proc. DARS'02. Japan.

Kansal, A., Somasundara, A., Jea, D., Srivasta, M., and Estrin, D. 2004. Intelligent fluid infrastructure for embedded networks. In Proc. ACM MOBISYS'04. Boston, MA.

Karlin, S. and Taylor, H. 1998. An Introduction to Stochastic Modeling, 3rd ed. Academic Press, Orlando, FL.

Karp, B. And Kung, H. 2000. Greedy perimeter stateless routing. In Proc. ACM MOBICOM'O0. Boston, MA.

Kumar, S., LAI, T., AND BALOGH, J. 2004. On k-coverage in a mostly sleeping sensor network. In Proc. ACM MOBICOM'04. Philadelphia, PA.

Liu, B., Brass, P., Dousse, O., Nain, P., And Towsley, D. 2005. Mobility improves coverage of sensor networks. In Proc. ACM MOBIHOC'05. Urbana-Champaign, IL.

Mondada, F., Franzi, E., And Ienne, P. 1993. Mobile robot miniaturisation: a tool for investigation in control algorithms. In Proc. of the Third International Symposium on Experimental Robotics (ISER'93). Kyoto, Japan.

Ross, S. 1989. Introduction to Probability Models, 4th ed. Academic Press, Boston, MA.

SChindelHauer, C. 2006. Mobility in wireless networks. In invited talk of SOFSEM'06. Merin, Czech Republic. 
Sibley, G., Rahimi, M., and Sukhatme, G. 2002. Robomote: A tiny mobile robot platform for large-scale sensor networks. In Proc. IEEE ICRA'02. Washington DC.

Slijepcevic, S. And Potkonjak, M. 2001. Power efficient organization of wireless sensor networks. In Proc. IEEE ICC'01. Helsinki, Finland.

WANG, D., Zhang, Q., AND LiU, J. 2007. The self-protection problem in wireless sensor networks. ACM Transactions on Sensor Networks 4 (Nov.).

Wang, G., CaO, G., and LaPorta, T. 2003. A bidding protocol for deploying mobile sensors. In Proc. IEEE ICNP'03. Atlanta, GA.

Xing, G., Lu, C., Pless, R., And O'Sullivan, J. 2004. Co-grid: an efficient coverage maintenance protocol for distributed sensor networks. In Proc. ACM IPSN'04. Berkeley, CA.

Xu, Y., Heidemann, J., and Estrin, D. 2001. Geography-informed energy conservation for ad-hoc routing. In Proc. ACM MOBICOM'01. Rome, Italy.

Yan, T., He, T., And Stankovic, J. 2003. Differentiated surveillance of sensor networks. In Proc. ACM SENSYS'03. Los Angeles, CA.

Ye, F., Zhong, G., Cheng, J., Lu, S., And Zhang, L. 2003. Peas: A robust energy conserving protocol for long-lived sensor networks. In Proc. IEEE ICDCS'03. Providence, RI.

Younis, O. And Fahmy, S. 2004. Distributed clustering in ad-hoc sensor networks: A hybrid, energy-efficient approach. In Proc. IEEE INFOCOM'04. Hong Kong, China.

Zou, Y. and Chakrabarty, K. 2003. Sensor deployment and target localizatoin based on virtual forces. In Proc. IEEE INFOCOM'03. San Francisco, CA.

Zou, Y. and Chakrabarty, K. 2004. Sensor deployment and target localization in distributed sensor networks. ACM Transactions on Embedded Computing Systems 1 (Feb.), 61-91. 